\title{
BMJ Open Association between right atrial area measured by echocardiography and prognosis among pulmonary arterial hypertension: a systematic review and meta-analysis
}

Ke Liu (D , ${ }^{1}$ Chunhua Zhang, ${ }^{2}$ Bingyu Chen, ${ }^{3}$ Mingfeng Li, ${ }^{4}$ Peican Zhang ${ }^{4}$

To cite: Liu K, Zhang C, Chen B, et al. Association between right atrial area measured by echocardiography and prognosis among pulmonary arterial hypertension: a systematic review and meta-analysis. BMJ Open 2020;10:e031316. doi:10.1136/ bmjopen-2019-031316

- Prepublication history and additional material for this paper is available online. To view these files, please visit the journal online (http://dx.doi.org/10. 1136/bmjopen-2019-031316)

$\mathrm{KL}$ and $\mathrm{CZ}$ contributed equally.

Received 30 April 2019

Revised 12 August 2020

Accepted 19 August 2020

\section{Check for updates}

\section{(C) Author(s) (or their} employer(s)) 2020. Re-use permitted under CC BY-NC. No commercial re-use. See rights and permissions. Published by BMJ.

${ }^{1}$ Department of General Surgery, Zhengzhou People's Hospital,

Zhengzhou, Henan, China

${ }^{2}$ Department of Emergency, Shangcai People's Hospital,

Shangcai, Henan, China

${ }^{3}$ Department of Internal medicine, Nanjing University of Traditional Chinese Medicine, Nanjing, China

${ }^{4}$ Department of Emergency, The First Affiliated Hospital of Henan University, Kaifeng, Henan, China

Correspondence to

Dr Ke Liu;

liukedoctor77@163.com

\section{ABSTRACT}

Objective The purpose of this meta-analysis was to evaluate the association between enlarged right atrial area (RAA), as measured by echocardiography, and prognosis of patients with pulmonary arterial hypertension (PAH). Design Systematic review and meta-analysis.

Data sources To identify potential publications, a comprehensive literature search through MEDLINE, the Cochrane database and the Embase database was performed up to December 2019.

Eligibility criteria for selecting studies Studies were included if they reported Cox regression basedHRs with $95 \%$ Cls for all-cause mortality or composite endpoint consisting of death and PAH-related events for echocardiography measurements of the RAA or the right atrial area index (RAAl) in patients with PAH.

Data extraction and synthesis The unadjusted HR with $95 \% \mathrm{Cl}$ was extracted for the final pooled analysis. A random-effects model was used to determine the value of RAA/RAAl in the prognosis of patients with PAH. The data heterogeneity among the studies was estimated by the $\mathrm{I}^{2}$ statistic and the Cochran Q-statistic.

Results Twelve studies with a total of 1085 patients with $\mathrm{PAH}$ were finally included in the meta-analysis. These studies had a mean follow-up time ranging from 9.2 months to 5.0 years. Their findings showed that patients with PAH with enlarged RAA/RAAl were associated with poor prognosis. The risk of all-cause mortality in patients with PAH was found to statistically increase by $50 \%$ for every 5-unit increase in RAA/RAAl (HR 1.50, 95\% Cl 1.28 to $1.75, p<0.001)$. Similarly, the risk of the composite endpoint also significantly increased by $53 \%$ for every 5-unit increase in RAA/RAAI (HR 1.53, 95\% Cl 1.23 to 1.89 , $\mathrm{p}<0.001)$. Subgroup analyses in which the patients were stratified by RAA and RAAl were consistent with the main results.

Conclusion The meta-analysis suggested that enlarged RAA/RAAl were associated with increased risk of poor prognosis in patients with $\mathrm{PAH}$.

\section{INTRODUCTION}

Pulmonary arterial hypertension $(\mathrm{PAH})$ is a pathophysiological disorder caused by the progressive narrowing of small pulmonary
Strengths and limitations of this study

- This meta-analysis is the largest study to date examining the association between right atrial area/ right atrial area index as measured by echocardiography and poor prognosis in patients with pulmonary arterial hypertension.

- The heterogeneity between included studies concerning the primary endpoint was relatively low, which enhanced the robustness of this meta-analysis.

- Only univariable HRs were pooled, due to the heterogeneous multivariable adjustment models used in the different studies.

- The studies included were observational, and mostly single centred, with small study populations.

arteries, which can lead to increased pulmonary vascular resistance and right-sided heart failure. ${ }^{12}$ Despite the availability of modern treatments, the disastrous prognosis (5-year mortality expectation approaching $30 \%-50 \%)$ of patients with PAH shows the urgent need for amelioration of this condition. $^{34}$ To achieve optimal clinical management for each patient, risk assessment plays a pivotal role in determining clinical practice by multidisciplinary teams. Current guidelines recommend categorising patients with PAH' risk of severity as low, intermediate or high on the basis of certain prognostic determinants to guide therapeutic decisions. ${ }^{2}$ Echocardiography is a well-established and widely available technique that is routinely used during the initial screening, follow-up assessment and evaluation of treatment responses for patients with $\mathrm{PAH}^{5-7}$ Despite advances in other imaging modality techniques such as MRI or CT, echocardiography remains a convenient and valuable option for assessing cardiac morphology and function. 
A number of echocardiographic parameters has been recently shown to be associated with prognosis in patients with severe $\mathrm{PAH}^{8}{ }^{9}$

At diagnosis, the right ventricular and right atrial areas (RAAs) are commonly increased in size. ${ }^{10}$ Theoretically, the right atrium (RA) is involved in the pathophysiological process of PAH. ${ }^{11}{ }^{12}$ Both right atrial reservoir and conduit functions are impaired and these impairments are associated with right ventricular (RV) pump dysfunction. ${ }^{13}$ An enlarged RAA, which reflects right ventricle overload in PAH, is recognised as a risk factor for adverse outcome. ${ }^{14}$ However, different studies have reported controversial results regarding the predictive value of this parameter, especially considering the imprecision of the echocardiographic measuring method. ${ }^{15} 16$ These various studies have reported a broad range of sample sizes, patient treatments, follow-up durations, outcome parameters and pulmonary hypertension $(\mathrm{PH})$ groups, yielding a challenge to establish the potential prognostic role of RAA in patients with PAH. Hence, the purpose of this systematic review and meta-analysis was to evaluate the association between RAA/right atrial area index (RAAI) measured by echocardiography and prognosis in patients with PAH.

\section{METHODS}

\section{Patient and public involvement statement}

Only the authors listed in this study were involved in the design and planning of the study, and neither the patients nor other members of the public influenced that process.

\section{Search strategy}

A comprehensive literature search through MEDLINE, the Cochrane database and the Embase database was performed according to the Preferred Reporting Items for Systematic Reviews and Meta-Analyses guidelines. ${ }^{17}$ To identify potential studies up to 1 December 2019, the following keywords were adopted: 'pulmonary hypertension', 'pulmonary arterial hypertension', 'echocardiography', 'right heart' 'right atrium', 'right atrial size', 'right atrial area', 'right atrial area index' and 'prognosis'. The details of the search strategy were presented in online supplemental file. The search was restricted to English-language publications. When several publications included the same group of patients (duplicate data), only that one with the most complete data or largest sample size was included. All of the eligible publications were evaluated independently for inclusion criteria by the two reviewers, and discordances were resolved by consensus with a third reviewer. The schematic representation of the article searching and screening process is presented in figure 1.

\section{Study selection criteria and endpoint definitions}

The included studies had to meet all of the following criteria: (1) designed as cohort studies; (2) investigated the effects of RAA or RAAI on all-cause mortality or

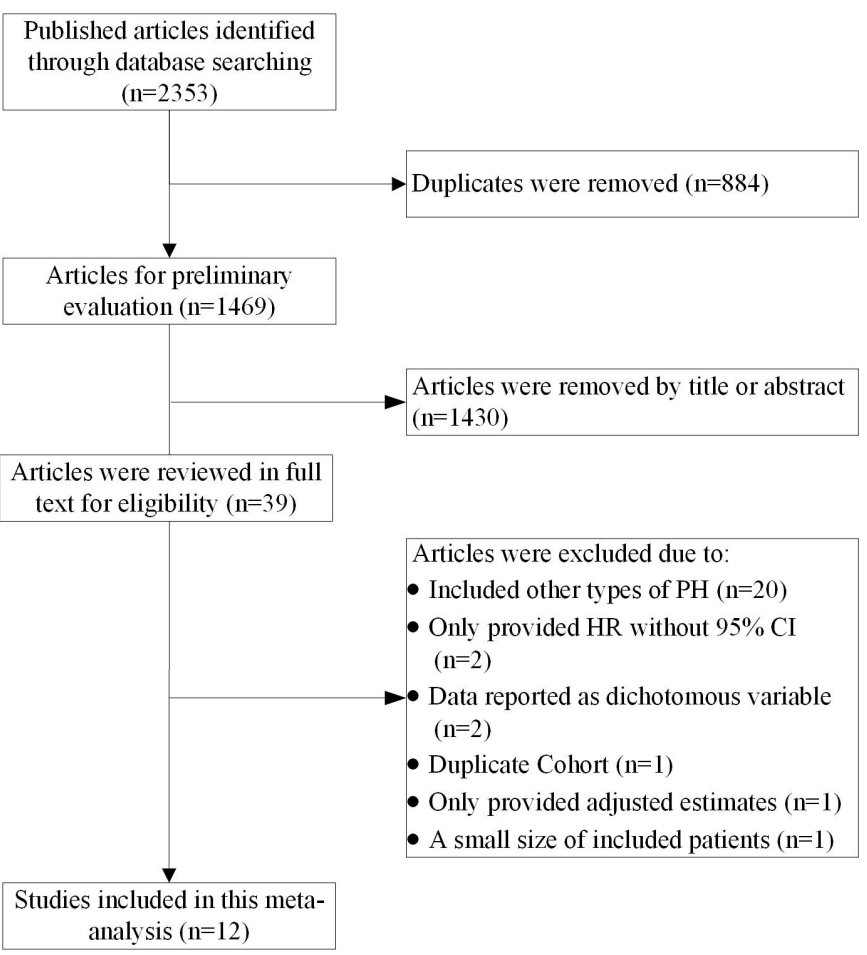

Figure 1 The schematic representation of article searching and screening. $\mathrm{PH}$, pulmonary hypertension.

the composite endpoint; (3) patients diagnosed with PAH according to standard guideline-based diagnostic criteria $^{18}$; (4) HRs with 95\% CIs reported using RAA or RAAI as the continuous variable; (5) sample sizes of no less than 40 patients.

The primary endpoint was all-cause mortality and the secondary endpoint was a composite endpoint of death and PAH-related events. PAH-related events were heterogeneous between the various studies, included lung transplantation, rehospitalisation due to worsening heart failure, initiation of prostanoids or need for new PAH drug treatment, and clinical worsening of a reduction in the 6 min walking test or WHO functional class.

\section{Data extraction and quality assessment}

The data were extracted independently by two investigators, and discrepancies were resolved by consensus with a third investigator. The following features of each eligible study were extracted using a standardised form: study design and population characteristics, PAH targeted therapies, echocardiographic findings, study endpoint and follow-up durations. The unadjusted HR with 95\% CI was extracted for the final pooled analysis. Initially, we harmonised group-level exposure estimates to per 5-unit changes, thereby allowing for integration of the estimated effects of RAA/RAAI differing in distinct studies. If the intensity of RAA/RAAI changing was not reported, we assumed that the intensity was one-unit change.

A dedicated tool designed for prognostic study assessment was adopted to evaluate the methodological quality of the included publications. ${ }^{19}{ }^{20}$ This tool consists of the following six methodological domains to assess potential 

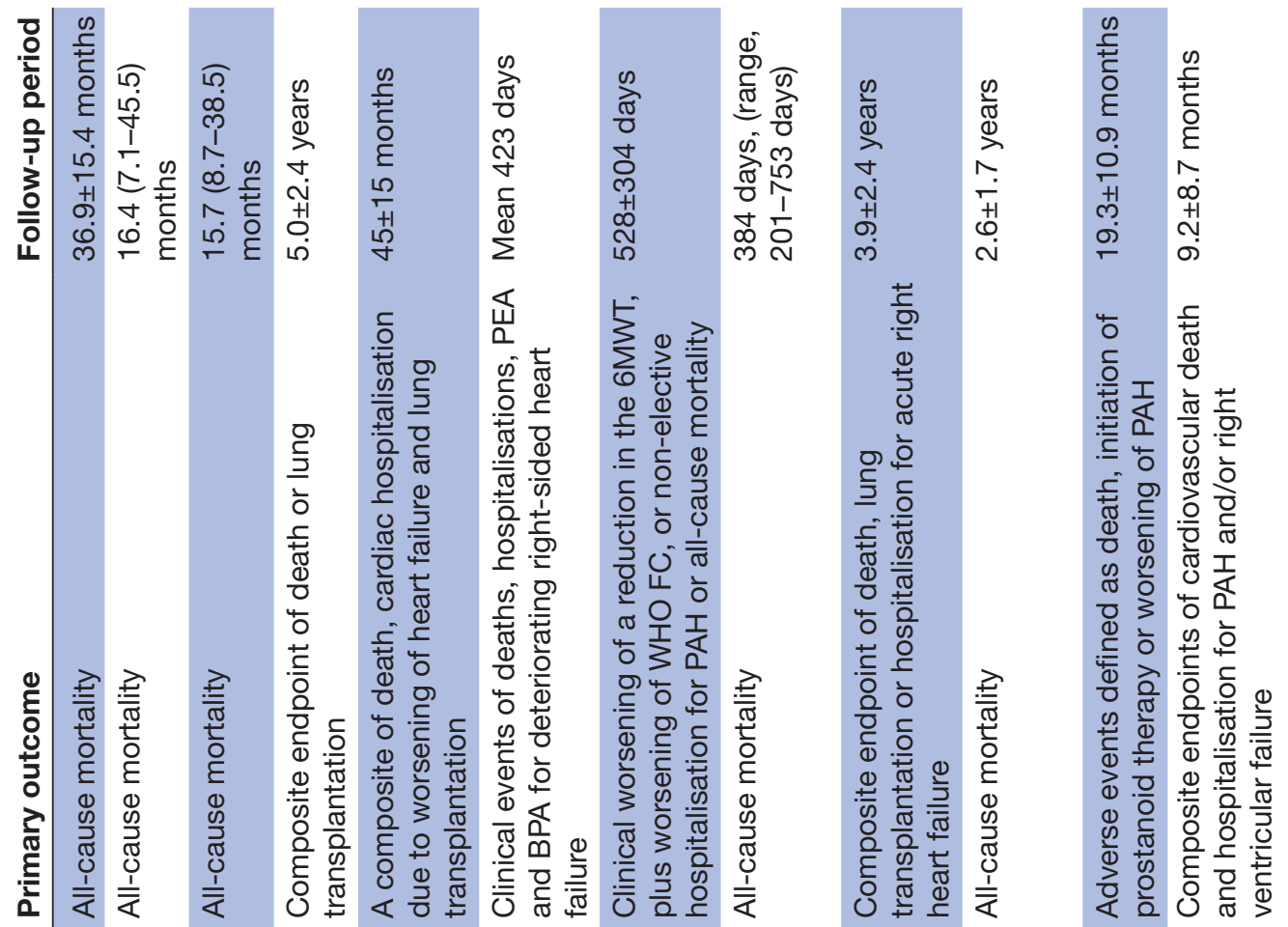

\begin{tabular}{l}
0 \\
2 \\
2 \\
0 \\
0 \\
\hline \\
\hline
\end{tabular}

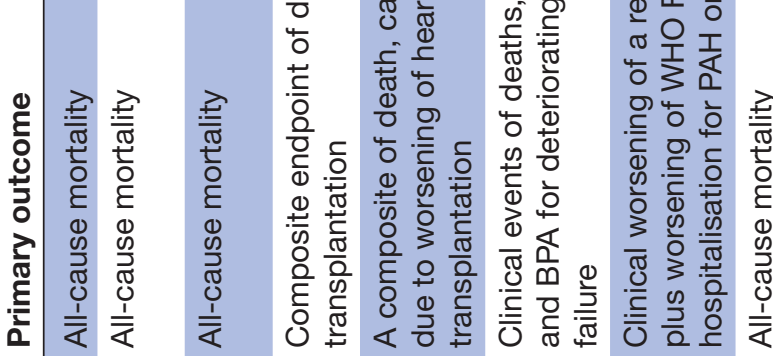

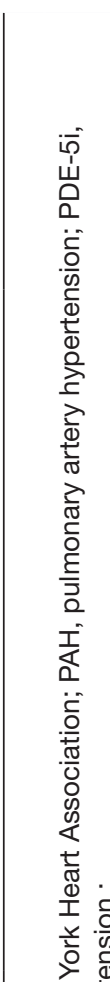

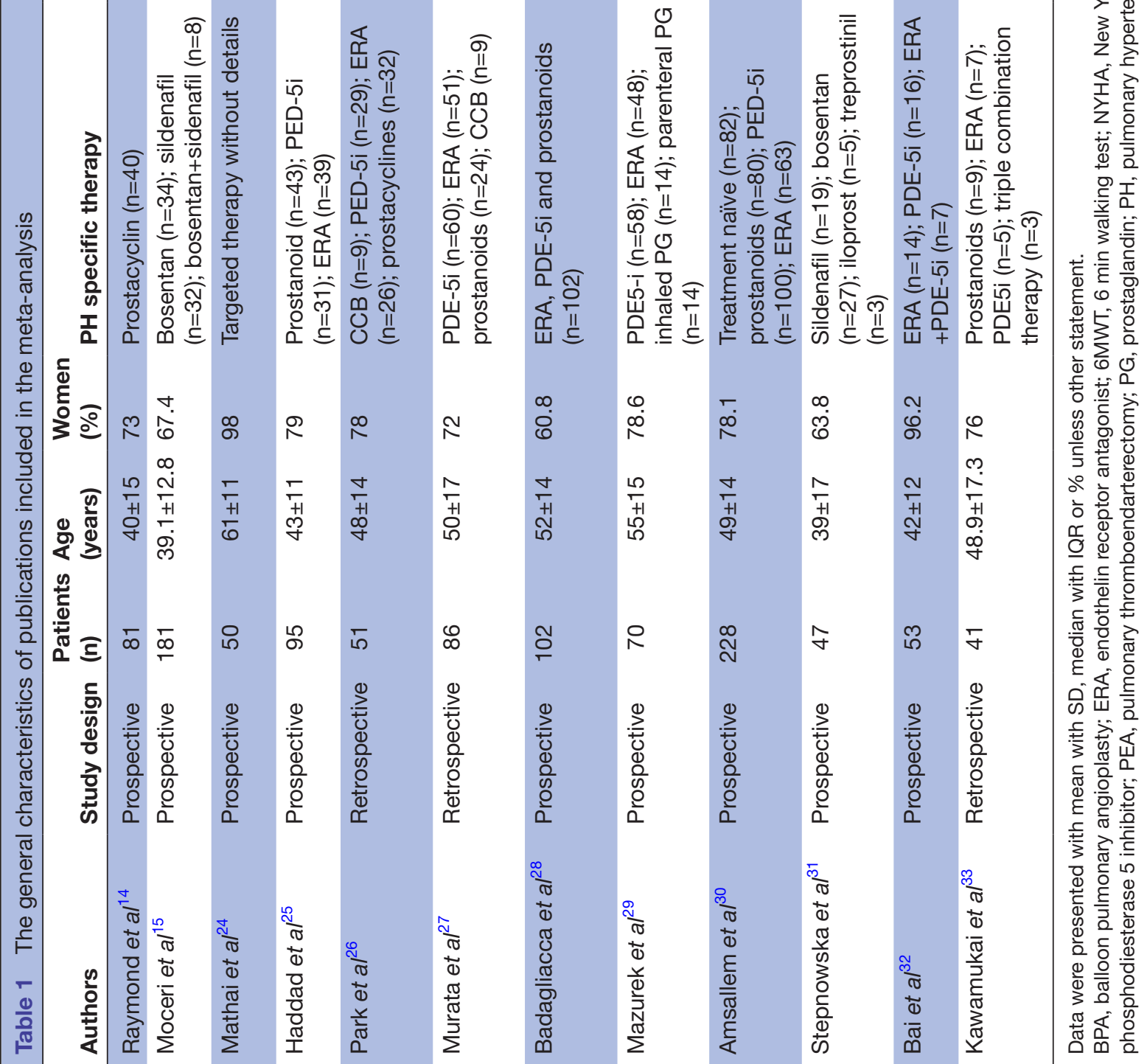


Table 2 Values of right atrial area or right atrial area index and $\mathrm{HR}$ with $95 \% \mathrm{Cl}$ from the publications included in the metaanalysis

\begin{tabular}{|c|c|c|c|c|c|c|}
\hline \multirow[b]{2}{*}{ Authors } & \multirow[b]{2}{*}{ Right atrial size } & \multirow[b]{2}{*}{$\begin{array}{l}\text { Changing } \\
\text { amplitude }\end{array}$} & \multicolumn{2}{|l|}{ Events (n) } & \multicolumn{2}{|l|}{ HR $(95 \% \mathrm{Cl})$} \\
\hline & & & $\begin{array}{l}\text { All-cause } \\
\text { mortality }\end{array}$ & $\begin{array}{l}\text { Composite } \\
\text { endpoint }\end{array}$ & All-cause mortality & $\begin{array}{l}\text { Composite } \\
\text { endpoint }\end{array}$ \\
\hline Moceri et $a l^{15}$ & $21.1 \pm 6.1$ & Per $10 \mathrm{~cm}^{2}$ & 19 & NA & 3.59 (1.92 to 6.72$)$ & \\
\hline Park et $\left.a\right|^{26}$ & $22.5 \pm 9.4$ & Per $9.4 \mathrm{~cm}^{2}$ & 12 & 20 & 1.36 (0.85 to 2.18$)$ & 1.45 (1.02 to 2.05$)$ \\
\hline Murata et $a l^{27}$ & $18 \pm 5$ & Per $1 \mathrm{~cm}^{2}$ & NA & 19 & NA & 1.20 (1.06 to 1.34$)$ \\
\hline Stepnowska et $\left.a\right|^{31}$ & $\begin{array}{l}29 \pm 11 \text { (died) vs } 19 \pm 6 \\
\text { (survival) }\end{array}$ & Per $1 \mathrm{~cm}^{2}$ & 9 & NA & 1.08 (1.02 to 1.14$)$ & NA \\
\hline Bai et $a l^{32}$ & $\begin{array}{l}28.2 \pm 7.3 \text { (with events) vs } \\
17.9 \pm 4.2 \text { (without events) }\end{array}$ & Per $1 \mathrm{~cm}^{2}$ & NA & 20 & NA & $1.13(1.08$ to 1.18$)$ \\
\hline Kawamukai et a/ ${ }^{33}$ & $\begin{array}{l}18.0 \pm 8.0 \text { (with events) vs } \\
19.8 \pm 6.9 \text { (without events) }\end{array}$ & Per $1 \mathrm{~cm}^{2}$ & NA & 18 & NA & 0.97 (0.90 to 1.02$)$ \\
\hline Mathai et $a l^{24}$ & $14.0 \pm 5.5$ & Per $1 \mathrm{~cm}^{2}$ & 25 & NA & 1.11 (1.02 to 1.19$)$ & NA \\
\hline Haddad et $a l^{25}$ & NA & Per $5 \mathrm{~cm}^{2}$ & NA & 27 & NA & 1.81 (1.44 to 2.28$)$ \\
\hline Mazurek et $\left.a\right|^{29}$ & $13.0 \pm 4.4$ & Per $1 \mathrm{~cm}^{2}$ & 18 & NA & 0.98 (0.62 to 1.56$)$ & NA \\
\hline Amsallem et $\left.a\right|^{30}$ & $12.1 \pm 4.7$ & Per $4.7 \mathrm{~cm}^{2}$ & NA & 88 & $\mathrm{NA}$ & $1.37(1.20$ to 1.57$)$ \\
\hline
\end{tabular}

NA, not available; RAA, right atrial area; RAAl, right atrial area index.

biases among included studies: study participation, study attrition, prognostic factor measurement, outcome measurement, confounding measurement and account and statistical analysis and reporting. After assessment, every methodological domain was labelled as good, adequate or unclear in each study.

\section{Statistical analysis}

STATA software (V.14.0; StatCorp) was used to perform statistical analysis. Separate analyses were conducted for (1) all-cause mortality and (2) composite endpoint

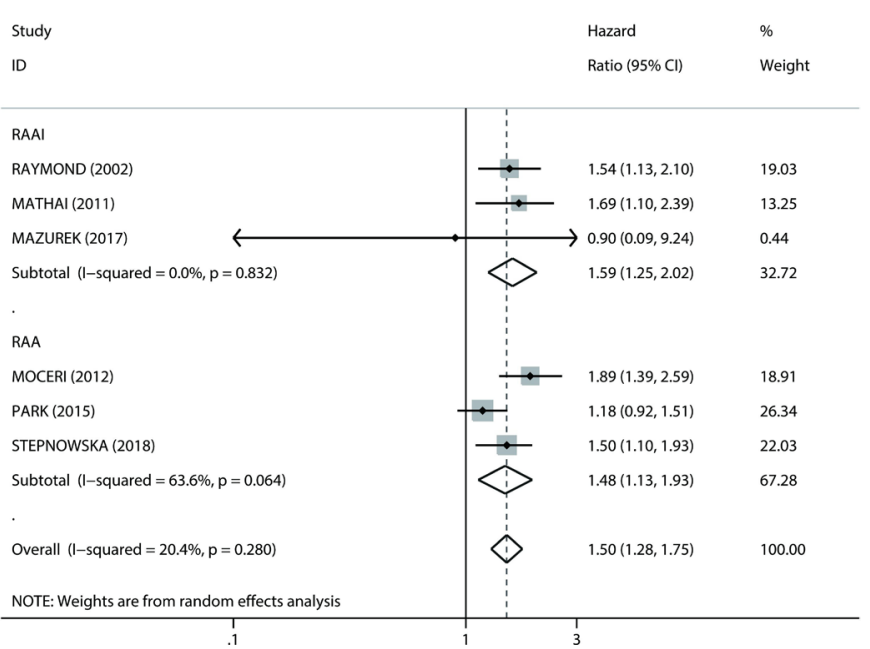

Figure 2 Forest plot comparing the unadjusted HRs of RAA/ RAAl for all-cause mortality pooled from included studies. RAA, right atrial area; RAAI, right atrial area index. of death and PAH-related events. The DerSimonia and Laird random effect models with the inverse varianceweighted mean of the logarithms of $\mathrm{HR}$ with $95 \% \mathrm{CI}$ are used to pool HR estimates. ${ }^{21}$ A two-sided $\mathrm{p}<0.05$ was considered statistically significant. Data heterogeneity among the studies was estimated by the $\mathrm{I}^{2}$ statistic and the Cochran Q-statistic, with $\mathrm{p}<0.10$ indicating significant heterogeneity. ${ }^{22}$ Sensitivity analysis was carried out using the leave-one-out approach and meta-regression was used to explore the potential moderators of effect size. Publication bias was assessed by the Egger's test using the effect size with upper and lower limits for each trial, with $\mathrm{p}<0.05$ considered statistically significant. ${ }^{23}$

\section{RESULTS}

\section{Search selection and study characteristics}

We totally identified 2353 publications by initial search strategy. After removing 884 duplicates, 1469 publications are remained for further assessment. Among them, 1186 were excluded after review of their titles and abstracts, and 39 publications were then considered for potential inclusion. Through carefully reading full text, 12 studies $^{141524-33}$ were finally included in the meta-analysis. The mean follow-up times of these studies ranged from 9.2 months to 5.0 years. In terms of study design, all of the 12 publications were cohort studies, with 9 prospective studies and 3 retrospective studies. Four studies only provided results on all-cause mortality, ${ }^{15} 242931$ and five studies only reported results for the composite 


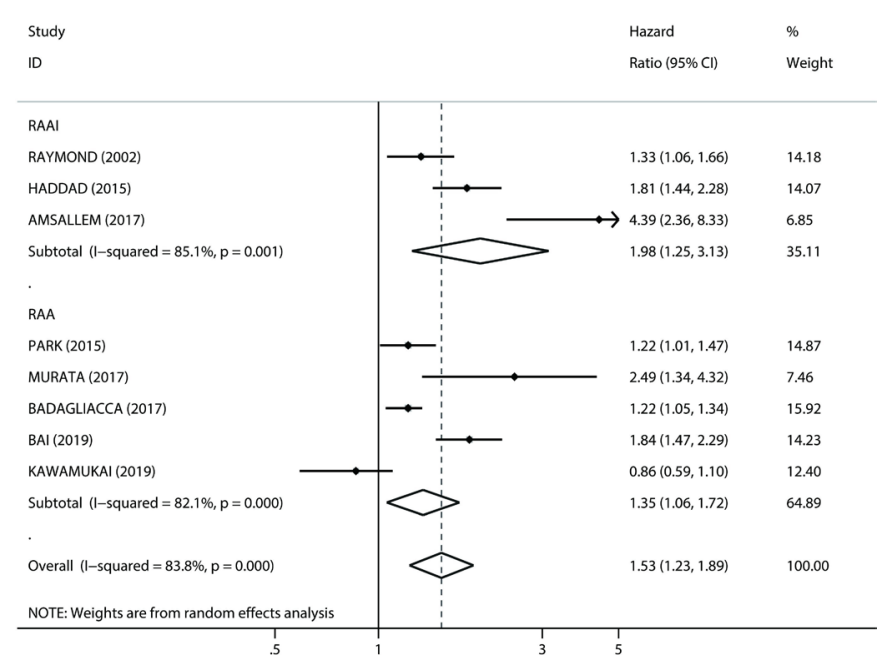

Figure 3 Forest plot comparing the unadjusted HRs of RAA/ RAAl for the composite point pooled from included studies. $\mathrm{RAA}$, right atrial area; RAAI, right atrial area index.

endpoint. $^{25} 27283233$ Two studies ${ }^{1426}$ reported separate data for all-cause mortality and composite endpoint, and therefore were eligible for both analysis.

\section{Patient characteristics}

A total of 1085 patients with PAH were included in this meta-analysis, of which female patients were predominant (ranging between $60.8 \%$ and $98.0 \%$ ), with mean age varying from 39 to 61 years. With respect to PAH targeted drugs therapies, the proportion of patients who received treatment and the types of drug used were fairly heterogeneous. Two publications reported the proportions of patients receiving a calcium channel blocker, 10 the proportion receiving a phosphodiesterase- 5 inhibitor and an endothelin receptor antagonist and 9 the proportion receiving a prostacyclin analogue. In the one remaining study, ${ }^{24}$ a demonstrated targeted therapy was prescribed, but no details were given. The key characteristics of the included studies are presented in table 1.

Table 2 summarised the echocardiographic measures of right atrial size (RAS) and the changes in numbers of units in the HRs referred to in included studies. The RAA was presented as an estimated parameter in eight studies, ${ }^{15}$ 26-28 30-33 whereas RAAI was presented in the other four studies. ${ }^{14242529}$ Moreover, all studies reported the numbers of events and the corresponding HR for primary and secondary endpoints.

\section{Endpoints and quality assessment}

The results revealed that the risk of all-cause mortality statistically increased by $50 \%$ for every 5 -unit increase in RAA/RAAI (HR 1.50, 95\% CI 1.28 to $1.75, \mathrm{p}<0.001$, see figure 2). Subgroup analyses showed the consistency between RAA (HR 1.48, 95\% CI 1.13 to $1.93, \mathrm{p}=0.004$ ) and RAAI (HR 1.59, 95\% CI 1.25 to 2.02 , p<0.001). Similarly, the risk of composite endpoint also significantly increased by $53 \%$ for every 5 -unit increase in RAA/RAAI (HR 1.53, 95\% CI 1.23 to $1.89, \mathrm{p}<0.001$, see figure 3 ). In addition, the pooled unadjusted HRs in different subgroups were in line with the main results, which was 1.35 in RAA (95\% CI 1.06 to $1.72, \mathrm{p}=0.016$ ) and 1.98 in RAAI (95\% CI 1.25 to $3.13, \mathrm{p}=0.003)$.

Quality assessment of included publications indicated a relatively low to moderate risk of bias. This assessment was performed using a QUIPS tool for prognostic studies, as described by Hayden et al. ${ }^{19}$ The results are presented in online supplemental table S1.

\section{Sensitivity analysis and publication bias}

Relatively low heterogeneity was found among the included studies with regard to the primary endpoint $\left(I^{2}=20.4 \%, p=0.280\right)$. Subgroup analyses showed that heterogeneity mainly originated from the RAA group. Sensitivity analysis by removing one study per time showed that the study by Park et al was as the source of heterogeneity. Exclusion of this study from the analysis markedly reduced the heterogeneity $\left(\mathrm{I}^{2}=0 \%, \mathrm{p}=0.805\right)$, and the pooled results were similar to the main finding. Similarly, we conducted sensitivity analysis at secondary endpoint, but we could not identify the key contributor to overall heterogeneity. Hence, possible association between the net effects of RAA/RAAI on the prognosis of patients with $\mathrm{PAH}$ and putative moderators (ie, the compositions of composite endpoint, study design, sample size, age, female proportion and duration of follow-up) were assessed using random effect meta-regression analysis. However, none covariates showed direct associations with the results $(\mathrm{p}>0.05)$.

Funnel plots with Egger's test showed no significant publication bias toward RAA/RAAI for primary endpoint and secondary endpoint, respectively (Egger's regression test $\mathrm{p}=0.169$ and $\mathrm{p}=0.262$, respectively) (see figure 4).

\section{DISCUSSION}

To date, our meta-analysis is the largest study of patients with PAH to examine the relationships between RAA/ RAAI and all-cause mortality, as well as the composite endpoint of death and PAH-related events. The main findings suggested that RAA/RAAI derived from echocardiography was significantly associated with the increased risk of poor prognosis in patients with $\mathrm{PAH}$.

To adequately assess the risk of patients with $\mathrm{PAH}$ for achieving more acceptable prognosis, accurate prognostication is highly important. Echocardiographic assessment of right ventricular longitudinal strain $^{26}$ and tricuspid annular plane systolic excursion ${ }^{34}$ have been established as important prognostic factors in determining disease severity and prognosis in patients with PAH. Nevertheless, only a few studies have investigated whether RAS or function were associated with prognosis for $\mathrm{PAH}$, partly due to morphological complexity of RA and the inconsistency of appraisal procedure. Regarding to RAS, BustamanteLabarta $e t a l^{35}$ first reported this parameter was an independent risk factor of transplantation and death in primary $\mathrm{PH}$, although with a small sample. Likely, Fukuda et a ${ }^{36}$ 

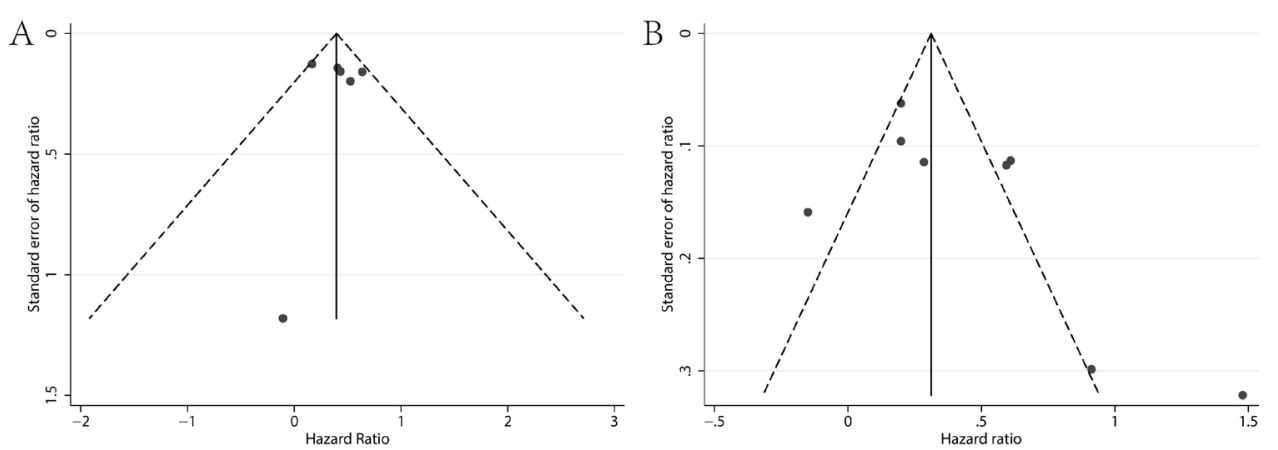

Figure 4 Funnel plot for all-cause mortality (A) and composite endpoint (B).

also found that combined assessment of impaired right ventricular systolic function and increased RAA resulted in more accurate prediction of long-term outcome.

Generally, the mechanics of the RA maintains cardiac function are complex. ${ }^{10}$ It has been recognised that the three components of atrial function are reservoir function, conduit function and contractile function. ${ }^{37} \mathrm{RA}$ is an anatomically dynamic structure, which is able to assist with filling of the RV at low pressure and responsible for up to $30 \%$ of normal RV output by contraction. RAS and pressure reflect right ventricular function. Gaynor et al demonstrated that the RA conduit-to-reservoir ratio is directly related to the $\mathrm{RV}$ pressure-RA pressure gradient. ${ }^{10}$ In contrast to primary left ventricular disease that causes elevation of left atrial pressure, PAH causes an elevation of right atrial pressure, which is inversely related to cardiac output. $^{38}$ In healthy subjects, short elevations of RV pressure lead to stretching and enlargement of the RA, resulting in higher reservoir volume. This mechanism has been identified as compensating for short-term RV overload. ${ }^{39}$ One significant observation has been that PAH is followed by enlargement and remodelling of the RA with hypertrophy and reduced contractility. ${ }^{40}$ However, right atrial compensation for the increased RV afterload has a limit. Once RA compensation for RV dysfunction is impaired, a scenario inevitably occurs that a decrease of cardiac output with the onset of severe right-side heart failure and rapid deterioration until death occurs. ${ }^{42} 43$ Additionally, right atrial enlargement due to increase in right atrial pressure could be a predisposing factor for the development of atrial arrhythmias, thus affecting the risk of hospitalisation and likely the prognosis in $\mathrm{PAH}^{4}{ }^{4}$

Currently, the algorithm of risk assessment of 2015 European Society of Cardiology(ESC)/European Respiratory Society(ERS) guidelines for the diagnosis and treatment of $\mathrm{PH},{ }^{2}$ suggests only one echo parameter, namely RAA, for use in multimarker risk stratification approach in patients with PAH. However, the evidence level of RAA for predicting prognosis in patients with PAH is low, and most importantly, the prognostic utility was only recommended for idiopathic PAH. ${ }^{2}$ Therefore, there is still insufficient solid evidence to demonstrate the prognostic value of RAA/RAAI for patients with PAH. The findings of this meta-analysis imply that enlarged RAA is a useful echocardiographic marker to predict mortality or composite endpoint in patients with $\mathrm{PAH}$, which is largely concordant with the guideline. Moreover, these results remind us that although other imaging examinations, such as MRI which may provide more accurate assessment of cardiac parameters, it is undeniable that echocardiography still has great advantages due to its easy availability and relatively cheapness.

Our meta-analysis revealed a lower capacity of RAA/ RAAI for predicting all-cause mortality and composite endpoint. This result may be explained partly by the fact that the studies included in the analysis provided the estimated effect of RAA/RAAI as a continuous variable. This analysis may underestimate the discriminating capacity of the parameters to predict prognosis in patients with a larger RAA. In addition, the composite endpoints in our meta-analysis were heterogeneous. Several studies have prespecified some clinical events, such as initiation of prostanoids or need for new PAH drug treatment, as a composite endpoint, which were objectively determined by physicians' consideration to a great extent. Accordingly, the diversity in clinical events consisting the composite endpoint might contribute to heterogeneity and reduce the capacity of RAA/RAAI to predict the risks of composite endpoint in patients with PAH.

This meta-analysis had several limitations. First, we presented only univariable HRs, because of the wide variety of multivariable adjustments between different studies (regarding the types and numbers of predictors per event used). Second, the included studies were observational, most of them involved a single centre with a small study population, and several were retrospective. Third, clinical inclusion and exclusion criteria used by the individual studies were variable and there was a wide variety of endpoints. This might explain the significant heterogeneity found between the included studies. Finally, we did not perform subgroup analysis based on ethnic groups in the selected studies due to insufficient data. Therefore, a complete picture of the impact of ethnicity on patients could not be obtained.

\section{CONCLUSION}

This present meta-analysis suggested that RAA/RAAI was associated with increased risk of poor prognosis in 
patients with PAH, as is currently indicated in the guidelines on RAA for the risk stratification of these patients.

Acknowledgements We greatly thank Ya-Cong Bo for assistance in data analysis and thank the professional copyediting services team (asiaedit) for improving the language.

Contributors $\mathrm{KL}$ and $\mathrm{CZ}$ designed the study and provided critical reviews of the manuscript. $\mathrm{BC}$ and $\mathrm{ML}$ reviewed the articles and extracted the data. $\mathrm{KL}$ and $\mathrm{CZ}$ conducted the data analysis. KL wrote the first draft of the manuscript and PZ critically revised it. All authors have reviewed and approved the final manuscript.

Funding The authors have not declared a specific grant for this research from any funding agency in the public, commercial or not-for-profit sectors.

Competing interests None declared.

Patient and public involvement Patients and/or the public were not involved in the design, or conduct, or reporting, or dissemination plans of this research.

Patient consent for publication Not required.

Provenance and peer review Not commissioned; externally peer reviewed.

Data availability statement All data relevant to the study are included in the article or uploaded as supplementary information. The datasets generated during and/or analysed during this study are available in the manuscript.

Open access This is an open access article distributed in accordance with the Creative Commons Attribution Non Commercial (CC BY-NC 4.0) license, which permits others to distribute, remix, adapt, build upon this work non-commercially, and license their derivative works on different terms, provided the original work is properly cited, appropriate credit is given, any changes made indicated, and the use is non-commercial. See: http://creativecommons.org/licenses/by-nc/4.0/.

ORCID iD

Ke Liu http://orcid.org/0000-0003-1450-910X

\section{REFERENCES}

1 Farber HW, Loscalzo J. Pulmonary arterial hypertension. N Engl J Med 2004;351:1655-65.

2 Galiè N, Humbert M, Vachiery J-L, et al. 2015 ESC/ERS guidelines for the diagnosis and treatment of pulmonary hypertension: the joint Task force for the diagnosis and treatment of pulmonary hypertension of the European Society of cardiology (ESC) and the European respiratory Society (ERS): endorsed by: association for European paediatric and congenital cardiology (AEPC), International Society for heart and lung transplantation (ISHLT). Eur Heart $J$ 2016;37:67-119.

3 Humbert M, Sitbon O, Chaouat A, et al. Survival in patients with idiopathic, familial, and anorexigen-associated pulmonary arterial hypertension in the modern management era. Circulation 2010;122:156-63.

4 Benza RL, Miller DP, Gomberg-Maitland M, et al. Predicting survival in pulmonary arterial hypertension: insights from the registry to evaluate early and long-term pulmonary arterial hypertension disease management (reveal). Circulation 2010;122:164-72.

5 Sachdev A, Villarraga HR, Frantz RP, et al. Right ventricular strain for prediction of survival in patients with pulmonary arterial hypertension. Chest 2011;139:1299-309.

6 Dimopoulos K, Condliffe R, Tulloh RMR, et al. Echocardiographic screening for pulmonary hypertension in congenital heart disease: JACC review topic of the week. J Am Coll Cardiol 2018;72:2778-88.

7 Mercurio V, Mukherjee M, Tedford RJ, et al. Improvement in right ventricular strain with ambrisentan and tadalafil upfront therapy in scleroderma-associated pulmonary arterial hypertension. Am J Respir Crit Care Med 2018;197:388-91.

8 Moceri P, Duchateau N, Baudouy D, et al. Three-dimensional right-ventricular regional deformation and survival in pulmonary hypertension. Eur Heart J Cardiovasc Imaging 2018;19:450-8.

9 Mukherjee M, Mercurio V, Tedford RJ, et al. Right ventricular longitudinal strain is diminished in systemic sclerosis compared with idiopathic pulmonary arterial hypertension. Eur Respir J 2017;50:1701436.

10 Gaynor SL, Maniar HS, Prasad SM, et al. Reservoir and conduit function of right atrium: impact on right ventricular filling and cardiac output. Am J Physiol Heart Circ Physiol 2005;288:H2140-5.

11 Sato T, Tsujino I, Oyama-Manabe N, et al. Right atrial volume and phasic function in pulmonary hypertension. Int $J$ Cardiol 2013;168:420-6.
12 Meng X, Li Y, Li H, et al. Three-dimensional echocardiography to evaluate right atrial volume and phasic function in pulmonary hypertension. Echocardiography 2018;35:153-61.

13 Querejeta Roca G, Campbell P, Claggett B, et al. Right atrial function in pulmonary arterial hypertension. Circ Cardiovasc Imaging 2015;8:e003521.

14 Raymond RJ, Hinderliter AL, Willis PW, et al. Echocardiographic predictors of adverse outcomes in primary pulmonary hypertension. J Am Coll Cardiol 2002;39:1214-9.

15 Moceri P, Dimopoulos K, Liodakis E, et al. Echocardiographic predictors of outcome in Eisenmenger syndrome. Circulation 2012;126:1461-8.

16 Siddiqui I, Rajagopal S, Brucker A, et al. Clinical and echocardiographic predictors of outcomes in patients with pulmonary hypertension. Am J Cardiol 2018;122:872-8.

17 Hutton B, Salanti G, Caldwell DM, et al. The PRISMA extension statement for reporting of systematic reviews incorporating network meta-analyses of health care interventions: checklist and explanations. Ann Intern Med 2015;162:777-84.

18 Hoeper MM, Bogaard HJ, Condliffe R, et al. Definitions and diagnosis of pulmonary hypertension. J Am Coll Cardiol 2013;62:D42-50.

19 Hayden JA, van der Windt DA, Cartwright JL, et al. Assessing bias in studies of prognostic factors. Ann Intern Med 2013;158:280-6.

20 Hayden JA, Côté P, Bombardier C. Evaluation of the quality of prognosis studies in systematic reviews. Ann Intern Med 2006;144:427-37.

21 DerSimonian R, Laird N. Meta-analysis in clinical trials revisited. Contemp Clin Trials 2015;45:139-45.

22 Higgins JPT, Thompson SG, Deeks JJ, et al. Measuring inconsistency in meta-analyses. BMJ 2003;327:557-60.

23 Egger M, Davey Smith G, Schneider M, et al. Bias in meta-analysis detected by a simple, graphical test. BMJ 1997;315:629-34.

24 Mathai SC, Sibley CT, Forfia PR, et al. Tricuspid annular plane systolic excursion is a robust outcome measure in systemic sclerosis-associated pulmonary arterial hypertension. $J$ Rheumatol 2011;38:2410-8.

25 Haddad F, Spruijt OA, Denault AY, et al. Right heart score for predicting outcome in idiopathic, familial, or drug- and toxinassociated pulmonary arterial hypertension. JACC CardiovasC Imaging 2015;8:627-38.

26 Park J-H, Park MM, Farha S, et al. Impaired global right ventricular longitudinal strain predicts long-term adverse outcomes in patients with pulmonary arterial hypertension. $J$ Cardiovasc Ultrasound 2015;23:91-9.

27 Murata M, Tsugu T, Kawakami T, et al. Prognostic value of threedimensional echocardiographic right ventricular ejection fraction in patients with pulmonary arterial hypertension. Oncotarget 2016;7:86781-90.

28 Badagliacca R, Papa S, Valli G, et al. Echocardiography combined with cardiopulmonary exercise testing for the prediction of outcome in idiopathic pulmonary arterial hypertension. Chest 2016;150:1313-22.

29 Mazurek JA, Vaidya A, Mathai SC, et al. Follow-up tricuspid annular plane systolic excursion predicts survival in pulmonary arterial hypertension. Pulm Circ 2017;7:361-71.

30 Amsallem M, Sweatt AJ, Aymami MC, et al. Right heart end-systolic remodeling index strongly predicts outcomes in pulmonary arterial hypertension: comparison with validated models. Circ Cardiovasc Imaging 2017;10:e005771.

31 Stepnowska E, Lewicka E, Dąbrowska-Kugacka A, et al. Predictors of poor outcome in patients with pulmonary arterial hypertension: a single center study. PLoS One 2018;13:e0193245.

32 Bai Y, Yang J, Liu J, et al. Right atrial function for the prediction of prognosis in connective tissue disease-associated pulmonary arterial hypertension: a study with two-dimensional speckle tracking. Int J Cardiovasc Imaging 2019;35:1637-49.

33 Kawamukai M, Hashimoto A, Koyama M, et al. Risk classification of pulmonary arterial hypertension by echocardiographic combined assessment of pulmonary vascular resistance and right ventricular function. Heart Vessels 2019;34:1789-800.

34 Forfia PR, Fisher MR, Mathai SC, et al. Tricuspid annular displacement predicts survival in pulmonary hypertension. $\mathrm{Am} \mathrm{J}$ Respir Crit Care Med 2006;174:1034-41.

35 Bustamante-Labarta M, Perrone S, De La Fuente RL, et al. Right atrial size and tricuspid regurgitation severity predict mortality or transplantation in primary pulmonary hypertension. J Am Soc Echocardiogr 2002;15:1160-4.

36 Fukuda $\mathrm{Y}$, Tanaka $\mathrm{H}$, Motoji $\mathrm{Y}$, et al. Utility of combining assessment of right ventricular function and right atrial remodeling as a prognostic factor for patients with pulmonary hypertension. Int $J$ Cardiovasc Imaging 2014;30:1269-77. 
37 D'Alto M, Scognamiglio G, Dimopoulos K, et al. Right heart and pulmonary vessels structure and function. Echocardiography 2015;32:S3-10.

38 Müller H, Reverdin S, Burri H, et al. Measurement of left and right atrial volume in patients undergoing ablation for atrial arrhythmias: comparison of a manual versus semiautomatic algorithm of real time 3D echocardiography. Echocardiography 2014;31:499-507.

39 Gaynor SL, Maniar HS, Bloch JB, et al. Right atrial and ventricular adaptation to chronic right ventricular pressure overload. Circulation 2005;112:I212-8.

40 Kushner FG, Lam W, Morganroth J. Apex sector echocardiography in evaluation of the right atrium in patients with mitral stenosis and atrial septal defect. Am J Cardiol 1978;42:733-7.
41 Cioffi G, de Simone G, Mureddu G, et al. Right atrial size and function in patients with pulmonary hypertension associated with disorders of respiratory system or hypoxemia. Eur J Echocardiogr 2007;8:322-31.

42 Bristow MR, Zisman LS, Lowes BD, et al. The pressure-overloaded right ventricle in pulmonary hypertension. Chest 1998;114:101S-6.

43 Ross J. Afterload mismatch and preload reserve: a conceptual framework for the analysis of ventricular function. Prog Cardiovasc Dis 1976;18:255-64.

44 Mercurio V, Peloquin G, Bourji KI, et al. Pulmonary arterial hypertension and atrial arrhythmias: incidence, risk factors, and clinical impact. Pulm Circ 2018;8:2045894018769874. 